New windows on massive stars: asteroseismology, interferometry, and spectropolarimetry

Proceedings IAU Symposium No. 307, 2014

G. Meynet, C. Georgy, J. H. Groh 8 Ph. Stee, eds.

(C) International Astronomical Union 2015 doi:10.1017/S1743921314007388

\title{
An X-ray surprise in a magnetic pulsator
}

\author{
Yaël Nazé \\ FNRS/ULg, Dept AGO, Allée du 6 Août 17, B5C, 4000-Liège, Belgium \\ email: naze@astro.ulg.ac.be
}

\begin{abstract}
CMa}$ is a rare $\beta$ Cep star with a strong magnetic field. To gain new insight on this object, a dedicated campaign using XMM-Newton was performed. These data reveal a new type of variations, X-ray pulsations, posing a new challenge to our understanding of stellar winds.
\end{abstract}

Keywords. stars: early-type, stars: oscillations, X-rays: stars, stars: individual ( $\left.\xi^{1} \mathrm{CMa}\right)$

\section{Introduction}

$\xi^{1} \mathrm{CMa}$ is a monoperiodic radial pulsator with a stable period and a strong magnetic field $(\sim 5 \mathrm{kG})$ - see Oskinova et al. (2014) and ref. therein. It was detected in the X-ray range by Einstein, and then observed by ROSAT and XMM (8ks snapshot in 2009): it is a moderately bright source $\left(F_{\mathrm{X}} \sim 10^{-12} \mathrm{erg} \mathrm{cm}^{-2} \mathrm{~s}^{-1}\right)$. It was reobserved with XMM during a long $(110 \mathrm{ks})$ run in Oct. $2012($ ObsID $=0691900101)$. The thick filter was used, to avoid contamination by opt/UV light. The XMM data were reduced in a standard way with SAS. Their full analysis is presented in Oskinova et al. (2014), here is discussed the timing analysis (my contribution to the latter paper, with some further details).

\section{The X-ray lightcurve}

Equivalent on-axis, full PSF lightcurves were extracted for $\xi^{1}$ CMa using SAS tasks (evselect, epiclccorr) for 3 time bins $(100,500,1000 \mathrm{~s})$ and 6 energy bands $(0.2-10$., $0.2-0.5,0.5-0.7,0.7-1 ., 0.2-1 ., 1 .-10 . \mathrm{keV})$. First, $\chi^{2}$ tests were performed for several hypotheses (constancy, linear variation, quadratic variation, Nazé et al. 2013): all pn lightcurves are significantly variable $(S L<0.01)$, as are the MOS1 lightcurves with 100s bins ; remaining MOS1 and MOS2 lightcurves show no significant variations. This difference comes from the many more counts in the pn data which allow more sensitive detections (see next section). Second, as the pn lightcurves in $0.2-10 \mathrm{keV}$ band seem to display a periodic signal (Fig. 1), a period search was performed. The calculation of the autocorrelation function led to the detection of $\mathrm{P}=17.1 \pm 1.5 \mathrm{ks}$, whereas a Fourier algorithm found a clear peak at $\mathrm{P}=17.6 \pm 0.3 \mathrm{ks}$ (Fig. 1). The spectral window is clean, a prewhitening by this period leaves only noise in the periodogram, and the false error probability associated to the peak is below $2 \times 10^{-8}$ : the period is therefore real. Finally, the best-fit sinusoid to the pn lightcurve in $0.2-10 \mathrm{keV}$ band was determined using $\chi^{2}$ (Fig. 1). It yields an amplitude of $0.034 \pm 0.004 \mathrm{ct} / \mathrm{s}$ (the same value as in the periodogram), corresponding to a pulsed fraction (=amplitude/mean) of $5.0 \pm 0.6 \%$, and a time $H J D_{0}$ for the maximum X-ray flux of $2456216.682 \pm 0.004$.

\section{Pulsations at different energies}

The Hipparcos catalog lists the following ephemeris for $\xi^{1} \mathrm{CMa}: \mathrm{P}=0.209577 \pm 0.000001 \mathrm{~d}$ and $J D_{0}=2448500.028 \pm 0.001$. This period corresponds to $18.10745 \pm 0.00009 \mathrm{ks}$ : the optical period is therefore fully compatible with the X-ray period (Oskinova et al. 2014). 

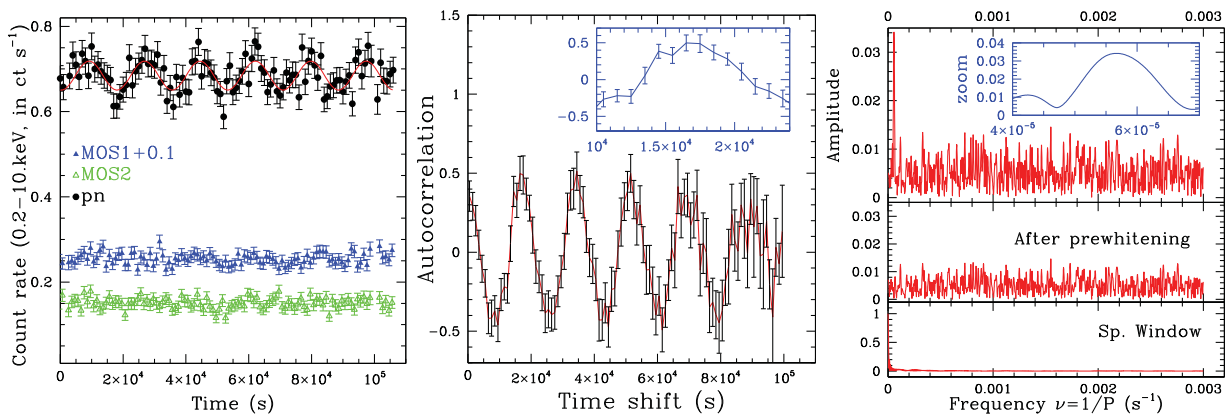

Figure 1. EPIC lightcurves of $\xi^{1} \mathrm{CMa}$, with best-fit sinusoid, autocorrelation, and periodogram for pn data.
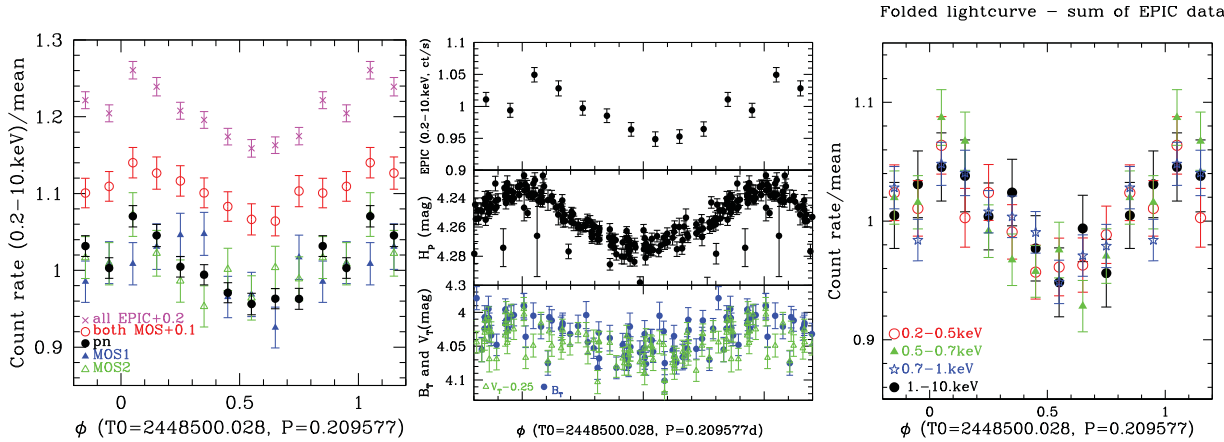

Figure 2. Folded pn, MOS, EPIC, and Hipparcos lightcurves.

The X-ray lightcurves were folded using these ephemeris (Fig. 2). The folded lightcurves of the individual and combined MOS are fully compatible with the presence of the periodic signal, both in amplitude and shape. There is thus no incompatibility between pn and MOS data. The folded lightcurves for the combined EPIC data yields a pulsed fraction of $4.1 \pm 0.5 \%$ in $0.2-10 . \mathrm{keV}$ energy band. Besides, the lightcurves clearly are energy-independent (Fig. 2). The Hipparcos lightcurve is very similar in shape to the combined EPIC lighcurve (Oskinova et al. 2014), but with a $3 \times$ lower amplitude (min$\max =0.037 \mathrm{mag}$, or a pulsed fraction of $1.73 \pm 0.07 \%$ in flux $)$. A small shift $(\sim 0.08$ in phase) is detected, but is not significant in view of the ephemeris uncertainties and the large number of cycles $(\sim 37000)$ between the observations.

\section{Conclusion}

X-ray variability has been detected in several massive stars: phase-locked changes in colliding-wind binaries, occultation effects in magnetically confined winds, and variations due to large-scale structures (e.g. CIRs) in single stars. A new type of variations, X-ray pulsations, is now discovered; their origin remains to be explained. Such simultaneous optical/X-ray variations were not seen in other pulsators (e.g. $\beta$ Cep, $\beta$ Cen) nor are predicted by theory (Oskinova et al. 2014).

\section{References}

Nazé, Y., Oskinova, L. M., \& Gosset, E. 2013, ApJ 763, 143

Oskinova, L. M., Nazé, Y., Todt, H., et al. 2014, Nature Communications 5, 4024 